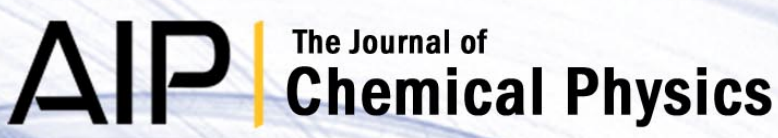

Ultracold $\mathrm{O} 2+\mathrm{O} 2$ collisions in a magnetic field: On the role of the potential energy surface

Jesús Pérez-Ríos, José Campos-Martínez, and Marta I. Hernández

Citation: J. Chem. Phys. 134, 124310 (2011); doi: 10.1063/1.3573968

View online: http://dx.doi.org/10.1063/1.3573968

View Table of Contents: http://jcp.aip.org/resource/1/JCPSA6/v134/i12

Published by the American Institute of Physics.

\section{Additional information on J. Chem. Phys.}

Journal Homepage: http://jcp.aip.org/

Journal Information: http://jcp.aip.org/about/about_the_journal

Top downloads: http://jcp.aip.org/features/most_downloaded

Information for Authors: http://jcp.aip.org/authors

\section{ADVERTISEMENT}

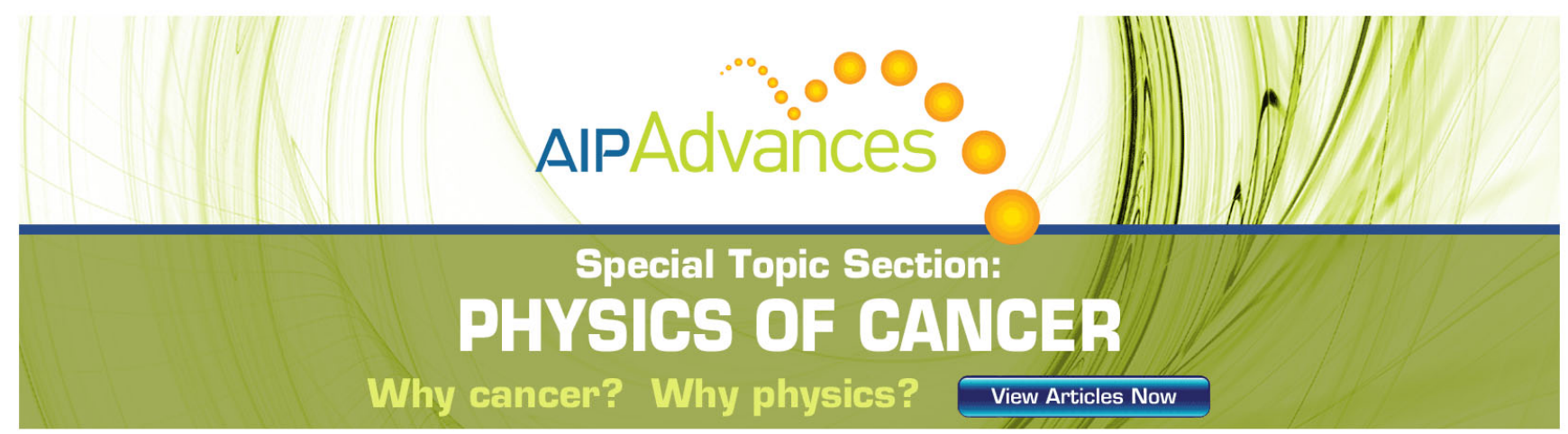




\title{
Ultracold $\mathrm{O}_{2}+\mathrm{O}_{2}$ collisions in a magnetic field: On the role of the potential energy surface
}

\author{
Jesús Pérez-Ríos, José Campos-Martínez, and Marta I. Hernández ${ }^{\text {a) }}$ \\ Instituto de Física Fundamental, Consejo Superior de Investigaciones Científicas (IFF-CSIC), Serrano 123, \\ 28006 Madrid, Spain
}

(Received 3 February 2011; accepted 15 March 2011; published online 29 March 2011)

\begin{abstract}
The collision dynamics of ${ }^{17} \mathrm{O}_{2}\left({ }^{3} \Sigma_{g}^{-}\right)+{ }^{17} \mathrm{O}_{2}\left({ }^{3} \Sigma_{g}^{-}\right)$in the presence of a magnetic field is studied within the close-coupling formalism in the range between $10 \mathrm{nK}$ and $50 \mathrm{mK}$. A recent global $a b$ initio potential energy surface (PES) is employed and its effect on the dynamics is analyzed and compared with previous calculations where an experimentally derived PES was used [T. V. Tscherbul et al., New J. Phys 134, 055021 (2009)]. Compared to the results using the older PES, magnetic-field dependence of the low-field-seeking state in the ultracold regime is characterized by a very large background scattering length, $a_{b g}$, and cross sections exhibit broader and more pronounced Feshbach resonances. The marked resonance structure is somewhat surprising considering the influence of inelastic scattering but it can be explained by resorting to the analytical van der Waals theory, where the short-range amplitude of the entrance channel wavefunction is enhanced by the large $a_{b g}$. This strong sensitivity to the short range of the $a b$ initio PES persists up to relatively high energies $(10 \mathrm{mK})$. After this study and despite quantitative predictions are very difficult, it can be concluded that the ratio between elastic and spin relaxation scattering is generally small, except for magnetic fields which are either low or close to an asymmetric Fano-type resonance. Some general trends found here, such as a large density of quasibound states and a propensity toward large scattering lengths, could be also characteristic of other anisotropic molecule-molecule systems. (C) 2011 American Institute of Physics. [doi:10.1063/1.3573968]
\end{abstract}

\section{INTRODUCTION}

Ultracold molecules play an important role in modern physics due to a large number of promising applications in quantum information, ${ }^{1}$ precision spectroscopy, ${ }^{2}$ and ultracold chemistry. ${ }^{3-5}$ Optical lattices of ultracold molecules are predicted to be ideally suited for quantum simulation of complex quantum systems ${ }^{6-8}$ and the engineering of new schemes for quantum information storage and processing. ${ }^{9,10}$ On the other hand, creation of a Bose-Einstein condensate (BEC) of molecules may enable studies of Bose-enhanced chemistry. ${ }^{11}$ In the context of these studies, molecules must be confined within a trap. For paramagnetic molecules, a magnetic trap is commonly used since molecules in a low-fieldseeking state ${ }^{12}(l f s)$ are trappable provided that their translational energy is lower than that of the trap depth. ${ }^{13}$ This situation could be achieved by direct cooling methods such as Zeeman slowing, ${ }^{14}$ optical Stark deceleration, ${ }^{15}$ singlephoton cooling, ${ }^{16}$ or sympathetic cooling. ${ }^{17}$ It might also be possible to cool the molecules toward the ultracold regime by evaporative cooling. ${ }^{18}$ As it is well known, this was the successful method for achievement of BEC of atoms. ${ }^{19,20}$

Molecular collisions are fundamental in this context, as evaporative cooling relies on efficient elastic collisions and, even more crucially, on the ratio $\gamma$ of the probabilities for elastic scattering and spin relaxation which must be very large in order to prevent heating and trap loss. External electromag-

\footnotetext{
a)Electronic mail: marta@iff.csic.es.
}

netic fields may serve to control the rate of inelastic collisions. Tuning close to a Feshbach resonance has proved to be an extremely fruitful means of controlling atom-atom collisions. ${ }^{21}$ Interestingly, it has been recently shown ${ }^{22}$ that inelastic collision rates in atom-molecule collisions can be tremendously reduced in the vicinity of a Feshbach resonance controlled by an electric or magnetic field.

While a large amount of work has been carried out for atom-atom and atom-molecule collisions, studies of molecule-molecule collisions in external fields are still scarce. Most clues about these more complex systems have come from atom-molecule studies. Krems and Dalgarno ${ }^{23}$ found that the main mechanisms of spin relaxation in collisions of ${ }^{3} \Sigma$ molecules with He is given by couplings to rotationally excited states mediated by the spin-spin interaction. Volpi and Bohn ${ }^{24}$ found that spin depolarization is suppressed when the Zeeman splitting between incident and final states does not exceed the height of the centrifugal barrier in the exit channel. These ideas were confirmed for ${ }^{17} \mathrm{O}_{2}\left({ }^{3} \Sigma_{g}^{-}\right)+$ ${ }^{17} \mathrm{O}_{2}\left({ }^{3} \Sigma_{g}^{-}\right)$by Tscherbul et al.,${ }^{25}$ who carried out the first accurate computational study involving two diatoms in a magnetic field. In that work, the experimentally derived potential energy surface (PES) of Aquilanti et al. ${ }^{26}$ was employed (Perugia PES in what follows). This collisional system is interesting since oxygen has been postulated as a reliable candidate for trapping and cooling ${ }^{27,28}$ and progress in cooling this species has been achieved recently. ${ }^{14,29}$

The present work builds up along these lines by the investigation of the role played by the PES in $\mathrm{O}_{2}+\mathrm{O}_{2}$ 
collisions in the presence of a magnetic field. It is well known that ultracold atom-atom collisions are very sensitive to the short range of the potential. ${ }^{30}$ However, it has been recently shown ${ }^{31}$ that, in the presence of inelastic scattering (i.e., atom-molecule collisions), peaks in cross sections around a Feshbach resonance may become suppressed and hence dynamics becomes rather insensitive to the details of the potential. This theory is tested here for a rather anisotropic molecule-molecule system, such as $\mathrm{O}_{2}+\mathrm{O}_{2}$, using a recent ab initio PES developed by Bartolomei et al. ${ }^{32}$ In this potential, electronic correlation is included by means of a high-level supermolecular method in the short range, whereas long-range interaction coefficients have been obtained from first principles as well. ${ }^{33}$ It is worthwhile to mention that inelastic rate coefficients obtained with this PES have proved to be highly consistent with measurements of the evolution of rotational populations along supersonic expansions in the temperature range $10 \leq T \leq 34 \mathrm{~K}^{34}$ By comparing present scattering calculations with previous ones using the Perugia PES (Ref. 25) and with some additional test modifications of the $a b$ initio PES, the effect of the potential on the cold and ultracold dynamics has been assessed.

This paper is organized as follows. In Sec. II, a summary of the theory for the scattering between two identical ${ }^{3} \Sigma$ molecules is given. Details specific to the ${ }^{17} \mathrm{O}_{2}-{ }^{17} \mathrm{O}_{2}$ system are provided in Sec. III and in Sec. IV, results are reported and discussed. A concluding remark is given in Sec. V.

\section{THEORY}

We give a summary of the theory-recently developed by Tscherbul et al. ${ }^{25}$-for the scattering of two ${ }^{3} \Sigma$ identical rigid rotor molecules in the presence of a magnetic field. Diatom-diatom Jacobi coordinates are used in a space-fixed (SF) frame, including the vector joining the centers of mass of the molecules $a$ and $b, \vec{R}$, and the intramolecular unit vectors, $\hat{r}_{a}$ and $\hat{r}_{b}$. Intramolecular distances are fixed to the molecular equilibrium distance, $r_{a}=r_{b}=r_{e}$. The Hamiltonian of the system can be written as

$$
\hat{H}=-\frac{1}{2 \mu R} \frac{\partial^{2}}{\partial R^{2}} R+\frac{\hat{l}^{2}}{2 \mu R^{2}}+V\left(\vec{R}, \hat{r}_{a}, \hat{r}_{b}\right)+\hat{H}_{a}+\hat{H}_{b},
$$

where atomic units are used $(\hbar=1), \hat{l}$ is the orbital angular momentum, $\mu$ is the reduced mass, and $V$ is the interaction potential or PES. The internal Hamiltonian of the ${ }^{3} \Sigma$ molecule $\hat{H}_{\alpha}(\alpha=a, b)$ is given, within the rigid rotor approximation, by ${ }^{35}$

$$
\begin{aligned}
\hat{H}_{\alpha}= & B_{e} \hat{n}_{\alpha}^{2}+2 \mu_{B} \vec{B} \cdot \hat{s}_{\alpha}+\gamma_{s r} \hat{n}_{\alpha} \cdot \hat{s}_{\alpha} \\
& +\frac{2}{3} \lambda_{s s} \sqrt{\frac{24 \pi}{5}} \sum_{q} Y_{2 q}^{*}\left(\hat{r}_{\alpha}\right)\left[\hat{s}_{\alpha} \otimes \hat{s}_{\alpha}\right]_{q}^{(2)},
\end{aligned}
$$

where $\hat{n}_{\alpha}$ is the angular momentum associated with $\hat{r}_{\alpha}, B_{e}$ is the rotational constant, $\mu_{B}$ is the Bohr magneton, $\vec{B}$ is the external magnetic field, and $\hat{s}$ is the electron spin. The last two terms in Eq. (2) correspond to the spin-rotation and spin-spin interactions, parameterized by $\gamma_{s r}$ and $\lambda_{s s}$, respec- tively. Hyperfine and intermolecular magnetic dipole interaction are neglected (see Ref. 28 for details). It is worth mentioning that, while the magnetic dipole interaction plays a very small role $(\approx 1 \%)$ in $\mathrm{O}_{2}+\mathrm{O}_{2}$ at zero magnetic field, ${ }^{28}$ it can be very important in other systems, as recently shown in Refs. 36 and 37.

The total wavefunction is expanded in a basis set of SF uncoupled and symmetry-adapted functions

$$
\Psi^{M \eta \epsilon}=\frac{1}{R} \sum_{\tau_{a} \geq \tau_{b} l m_{l}} u_{\tau_{a} \tau_{b} l m_{l}}^{M \eta \epsilon}(R) \phi_{\tau_{a} \tau_{b} l m_{l}}^{M \eta \epsilon}\left(\hat{R}, \hat{r}_{a} \hat{r}_{b}\right),
$$

with

$$
\phi_{\tau_{a} \tau_{b} l m_{l}}^{M \eta \epsilon}=\frac{1}{\left(2\left(1+\delta_{\tau_{a}, \tau_{b}}\right)\right)^{1 / 2}}\left(\left|\tau_{a} \tau_{b}\right\rangle+\eta \epsilon\left|\tau_{b} \tau_{a}\right\rangle\right)\left|l m_{l}\right\rangle,
$$

$\left|l m_{l}\right\rangle$ being a spherical harmonics and where $\left|\tau_{\alpha}\right\rangle$ represents an uncoupled function of the $\alpha$ monomer

$$
\left|\tau_{\alpha}\right\rangle=\left|n_{\alpha} m_{n_{\alpha}}\right\rangle\left|s_{\alpha} m_{s_{\alpha}}\right\rangle .
$$

The basis of Eq. (4) are a well-ordered set with $\tau_{a} \geq \tau_{b}$ that are normalized eigenfunctions of the operator permuting the identical molecular skeletons $\left(\hat{P}: \hat{r}_{a} \rightarrow \hat{r}_{b} ; \hat{r}_{b} \rightarrow \hat{r}_{a}\right.$; $\vec{R} \rightarrow-\vec{R}$ ), with eigenvalue $\eta$. These basis functions are also eigenfunctions of spatial inversion $\left(E^{*}: \hat{r}_{a} \rightarrow-\hat{r}_{a} ; \hat{r}_{b}\right.$ $\left.\rightarrow-\hat{r}_{b} ; \vec{R} \rightarrow-\vec{R}\right)$ with eigenvalue $\epsilon=(-1)^{n_{a}+n_{b}+l}$. Since the molecules under study are homonuclear, $n_{a}$ and $n_{b}$ have the same parity so $\epsilon=(-1)^{l}$. In addition to these symmetries, the Hamiltonian commutes with the SF $z$ axis component of the total angular momentum, so that for a given value of this projection, $M$, basis functions in Eq. (3) must satisfy

$$
m_{n_{a}}+m_{s_{a}}+m_{n_{a}}+m_{s_{a}}+m_{l}=M .
$$

Substitution of Eq. (3) into the Schrödinger equation leads to the set of close-coupled equations for the radial coefficients:

$$
\begin{aligned}
& {\left[\frac{1}{2 \mu} \frac{d^{2}}{d R^{2}}-\frac{l(l+1)}{2 \mu R^{2}}+E\right] u_{\tau_{a} \tau_{b} l m_{l}}^{M \eta \epsilon}(R)} \\
& \quad=\sum_{\tau_{a}^{\prime} \geq \tau_{b}^{\prime} l^{\prime} m_{l}^{\prime}}\left\langle\phi_{\tau_{a} \tau_{b} l m_{l}}^{M \eta \epsilon}\left|\left(V+\hat{H}_{a}+\hat{H}_{b}\right)\right| \phi_{\tau_{a}^{\prime} \tau_{b}^{\prime} l^{\prime} m_{l}^{\prime}}^{M \eta \epsilon} u_{\tau_{a}^{\prime} \tau_{b}^{\prime} l^{\prime} m_{l}^{\prime}}^{M \eta \epsilon}(R),\right.
\end{aligned}
$$

where $E$ is the total energy. It must be pointed out that the asymptotic Hamiltonian $\hat{H}_{a}+\hat{H}_{b}$ is not diagonal in the basis $\phi_{\tau_{a}^{\prime} \tau_{b}^{\prime} l^{\prime} m_{l}^{\prime}}^{M \eta \epsilon}$ due to the spin-rotation and spin-spin terms, and matrix elements of these terms are given in Eqs. (14) and (16) of Ref. 23, respectively. On the other hand, potential matrix elements are given as a sum of a direct and an exchange coupling terms: $:^{25}$

$$
\begin{aligned}
\left\langle\phi_{\tau_{a} \tau_{b} l m_{l}}^{M \eta \epsilon}|V| \phi_{\tau_{a}^{\prime} \tau_{b}^{\prime} l^{\prime} m_{l}^{\prime}}^{M \eta \epsilon}\right\rangle= & \frac{1}{\left[\left(1+\delta_{\tau_{a}, \tau_{b}}\right)\left(1+\delta_{\tau^{\prime}, \tau_{b}^{\prime}}\right)\right]^{1 / 2}} \\
& \times\left[\left\langle\tau_{a} \tau_{b} l m_{l}|V| \tau_{a}^{\prime} \tau_{b}^{\prime} l^{\prime} m_{l}^{\prime}\right\rangle\right. \\
& \left.+\eta \epsilon\left\langle\tau_{a} \tau_{b} l m_{l}|V| \tau_{b}^{\prime} \tau_{a}^{\prime} l^{\prime} m_{l}^{\prime}\right\rangle\right] .
\end{aligned}
$$

The interaction potential depends on the total spin resulting from the coupling of the $s_{a}=s_{b}=1$ spins of the ${ }^{3} \Sigma$ 
molecules, $S=0,1,2$, and can be represented as ${ }^{38}$

$$
V\left(\vec{R}, \hat{r}_{a}, \hat{r}_{b}\right)=\sum_{S=0}^{2} \sum_{M_{S}=-S}^{S} V_{S}\left(\vec{R}, \hat{r}_{a}, \hat{r}_{b}\right)\left|S M_{S}\right\rangle\left\langle S M_{S}\right|,
$$

where $M_{S}$ is the projection of the total spin, $M_{S}=m_{s_{a}}+m_{s_{b}}$. We use this representation in order to include directly the singlet, triplet, and quintet ab initio PESs of Ref. 32 (an alternative approach was followed in Ref. 25 since the Perugia PES is given as a sum of a spin-independent and a spin-dependent contribution $^{26}$ ). In this way, matrix elements of Eq. (8) can be further developed as

$$
\begin{aligned}
\left\langle\tau_{a} \tau_{b} l m_{l}|V| \tau_{a}^{\prime} \tau_{b}^{\prime} l^{\prime} m_{l}^{\prime}\right\rangle= & \delta_{M_{S}, M_{S}^{\prime}} \sum_{S=0}^{2}(2 S+1)\left(\begin{array}{ccc}
1 & 1 & S \\
m_{s_{a}} & m_{s_{b}} & -M_{S}
\end{array}\right) \\
& \times\left(\begin{array}{ccc}
1 & 1 & S \\
m_{s_{a}^{\prime}} & m_{s_{b}^{\prime}} & -M_{S}^{\prime}
\end{array}\right)\left\langle n_{a} m_{n_{a}} n_{b} m_{n_{b}} l m_{l}\right. \\
& \left.\times\left|V_{S}\right| n_{a}^{\prime} m_{n_{a}^{\prime}} n_{b}^{\prime} m_{n_{b}^{\prime}} l^{\prime} m_{l^{\prime}}\right\rangle,
\end{aligned}
$$

where (:::) are 3-j symbols. An explicit expression for $\left\langle n_{a} m_{n_{a}} n_{b} m_{n_{b}} l m_{l}\left|V_{S}\right| n_{a}^{\prime} m_{n_{a}^{\prime}} n_{b}^{\prime} m_{n_{b}^{\prime}} l^{\prime} m_{l^{\prime}}\right\rangle$ is given in Eq. (18) of Ref. 25.

Close-coupled equations [Eq. (7)] are solved by means a $\log$-derivative method ${ }^{39,40}$ and using the basis set of Eq. (4) in which, as mentioned above, the asymptotic Hamiltonian is not diagonal. In order to set scattering boundary conditions and thus, obtain the scattering $S$-matrix, it is necessary to transform to a new basis set $\psi_{\zeta_{a} \zeta_{b}, l, m_{l}}^{\eta}$ giving the eigenstates of the fragments. For each $l, m_{l}$ block:

$$
\left[\hat{H}_{a}+\hat{H}_{b}\right] \psi_{\zeta_{a} \zeta_{b} l m_{l}}^{M \eta \epsilon}=\left(\varepsilon_{\zeta_{a}}+\varepsilon_{\zeta_{b}}\right) \psi_{\zeta_{a} \zeta_{b} l m_{l}}^{M \eta \epsilon},
$$

where $\varepsilon_{\zeta_{\alpha}}$ is the Zeeman fine structure energy level of molecule $\alpha$. A unitary transformation of the log-derivative matrix onto the new basis is performed at the end of the propagation, and then scattering $S$-matrices and transition $T$ matrices are obtained in a standard way. ${ }^{25}$ The integral cross section for a transition $\zeta_{a} \zeta_{b} \rightarrow \zeta_{a}^{\prime} \zeta_{b}^{\prime}$ within a given $(M, \eta, \epsilon)$ block is finally given as

$$
\sigma_{\zeta_{a} \zeta_{b} \rightarrow \zeta_{a}^{\prime} \zeta_{b}^{\prime}}^{M \eta \epsilon}=\frac{\pi\left(1+\delta_{\zeta_{a}, \zeta_{b}}\right)}{k_{\zeta_{a} \zeta_{b}}^{2}} \sum_{l m_{l} l^{\prime} m_{l^{\prime}}}\left|T_{\zeta_{a} \zeta_{b} l m_{l} ; \zeta_{a}^{\prime} \zeta_{b}^{\prime} l^{\prime} m_{l^{\prime}}}^{M \eta \epsilon}\right|^{2}
$$

where $T$ is the transition matrix and $k_{\zeta_{a} \zeta_{b}}^{2} /(2 \mu)=E-\varepsilon_{\zeta_{a}}-$ $\varepsilon_{\zeta_{b}}$ is the translational energy of the initial channel. In obtaining Eq. (12), integration of the differential cross section has been restricted over half-space for final states satisfying $\zeta_{a}^{\prime}=\zeta_{b}^{\prime}$ (see Ref. 25). This is equivalent to dividing the cross sections integrated over full-space by two to avoid double counting when the state of the outgoing molecules is the same. ${ }^{41,42}$

\section{COMPUTATION DETAILS}

We consider collisions between ${ }^{17} \mathrm{O}_{2}\left({ }^{3} \Sigma_{g}^{-}\right)$identical molecules as in the work of Tscherbul et al. ${ }^{25}$ The ${ }^{17} \mathrm{O}$ isotope was chosen because field-free calculations of Avdeenkov and Bohn showed that ${ }^{17} \mathrm{O}_{2}$ is a much better candidate for ultracold studies than the more abundant ${ }^{16} \mathrm{O}_{2}$ isotopologue. ${ }^{28}$ The asymptotic Hamiltonian of Eq. (2) is parameterized by
TABLE I. Energies (in K) and coefficients [in the basis of Eq. (5)] of the three lowest states of ${ }^{17} \mathrm{O}_{2}$ [Eq. (2)] for a magnetic field $B=100 \mathrm{G}$.

\begin{tabular}{lrrrrr}
\hline \hline & $\varepsilon_{\zeta}(\mathrm{K})$ & \multicolumn{1}{c}{$C_{\tau \zeta}$} & $\mid n$ & $m_{n}$ & $\left.m_{s}\right\rangle$ \\
\hline$\zeta=1$ & -0.7035 & 0.9687 & $\mid 0$ & 0 & $-1\rangle$ \\
& & -0.1922 & $\mid 2$ & -2 & $1\rangle$ \\
& & 0.1361 & $\mid 2$ & -1 & $0\rangle$ \\
& & -0.0786 & $\mid 2$ & 0 & $-1\rangle$ \\
$\zeta=2$ & -0.6913 & 0.9686 & $\mid 0$ & 0 & $0\rangle$ \\
& & -0.1361 & $\mid 2$ & -1 & $1\rangle$ \\
& & 0.1573 & $\mid 2$ & 0 & $0\rangle$ \\
$\zeta=3$ & -0.6791 & 0.9684 & $\mid 0$ & 0 & $1\rangle$ \\
& & -0.0788 & $\mid 2$ & 0 & $1\rangle$ \\
& & 0.1364 & $\mid 2$ & 1 & $0\rangle$ \\
& & -0.1931 & $\mid 2$ & 2 & $-1\rangle$ \\
\hline \hline
\end{tabular}

means of accurate ${ }^{17} \mathrm{O}_{2}$ spectroscopic constants: ${ }^{43} B_{e}=1.353$ $\mathrm{cm}^{-1}, \gamma_{s r}=-0.00396 \mathrm{~cm}^{-1}$, and $\lambda_{s s}=1.985 \mathrm{~cm}^{-1}$. The three lowest states of the $n=$ even manifold (compatible with molecules in the stretched nuclear spin state $M_{I}=I=5$ [Ref. 28]) are given in Table I for a typical value of the magnetic field. Dependence with magnetic field of the combined $\left|\zeta_{a}, \zeta_{b}\right\rangle$ asymptotic states is depicted in Fig. 1. In this work, we focus on the initial state $\left|\zeta_{a}, \zeta_{b}\right\rangle=|3,3\rangle$, i.e., both molecules are, prior to interaction, in their lowest $l f s$ state. Elastic and inelastic integral cross sections are obtained for translational energies ranging from $10^{-8}$ to $0.05 \mathrm{~K}$. As we are dealing with collisions between identical (composite) bosons, calculations are restricted to the $\eta=+1$ block (the role of nuclear spin can be ignored, as explained in detail in Ref. 28). Note also that to study processes involving identical internal states [Eq. (4)], calculations are constrained to the $\epsilon=+1$ parity (only even $l$ 's in the wavefunction expansion).

The intermolecular interaction is given by the global $a b$ initio PES of Bartolomei et al., ${ }^{32}$ specifically, the one referred in that work as CC-PT2 PES. Singlet, triplet, and quintet $(S=0,1,2)$ potentials are given ${ }^{32}$ by the spherical harmonic

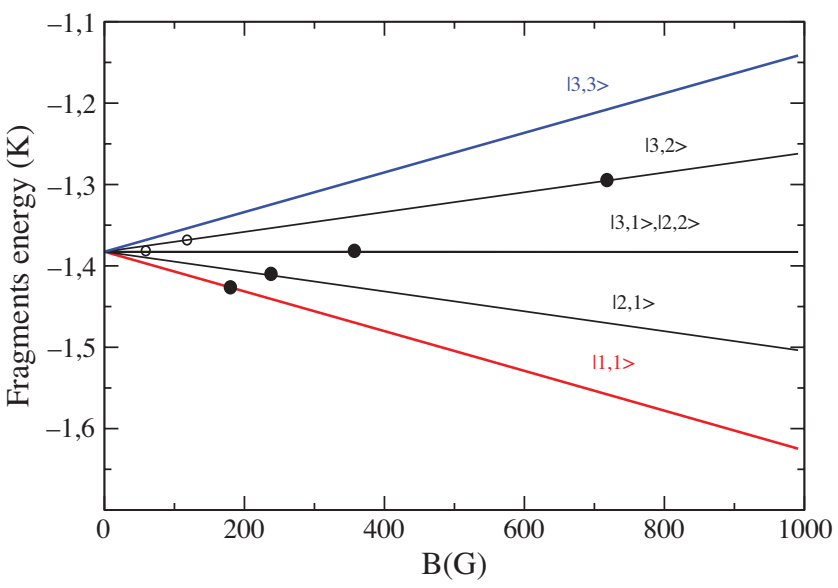

FIG. 1. Internal energies of ${ }^{17} \mathrm{O}_{2}\left(\zeta_{a}\right)+{ }^{17} \mathrm{O}_{2}\left(\zeta_{b}\right)$ as functions of magnetic field. In this work, molecules are considered to be initially in their lf $s$ states $\left|\zeta_{a}, \zeta_{b}\right\rangle=|3,3\rangle$. Open and closed symbols indicate critical values of the field for which $d$ and $g$ barriers, respectively, become open for the different outgoing channels. Note also that calculations of Fig. 4 refer to the $h f s$ state $|1,1\rangle$. 


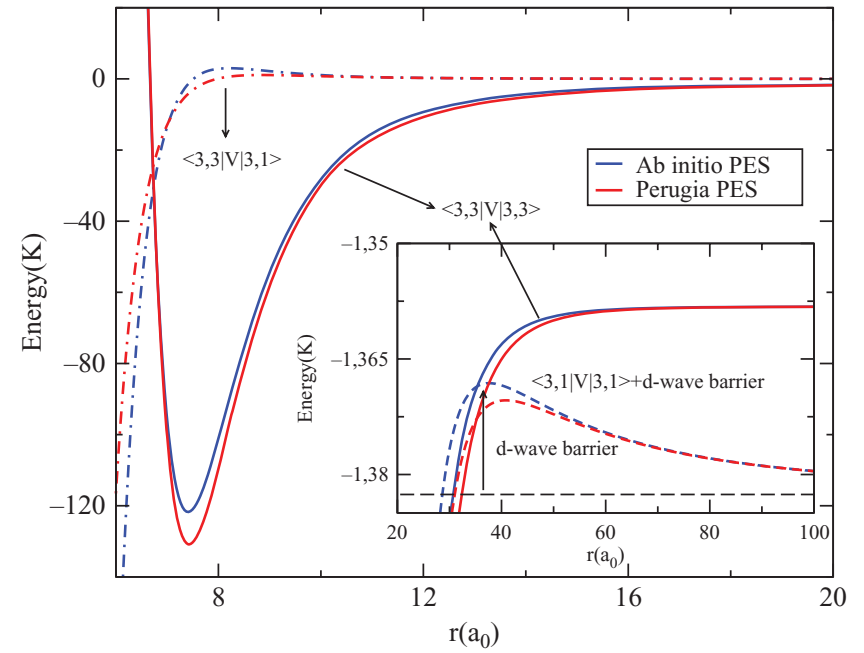

FIG. 2. Comparison of the $a b$ initio and Perugia potential matrix elements among the fragment states $|3,3\rangle$ and $|3,1\rangle$ for a magnetic field $B=100 \mathrm{G}$. The long-range behavior is compared in the inset. Note that orbital angular momentum for the entrance $(|3,3\rangle)$ and outgoing $(|3,1\rangle)$ channels are 0 and 2 , respectively.

expansion,

$$
V_{S}\left(\vec{R}, \hat{r}_{a}, \hat{r}_{b}\right)=(4 \pi)^{3 / 2} \sum_{\lambda_{a} \lambda_{b} \lambda} V_{S}^{\lambda_{a} \lambda_{b} \lambda}(R) A_{\lambda_{a} \lambda_{b} \lambda}\left(\hat{R}, \hat{r}_{a}, \hat{r}_{b}\right),
$$

where $A_{\lambda_{a} \lambda_{b} \lambda}$ is given as a combination of spherical harmonics and $\lambda_{a}, \lambda_{b}$, and $\lambda$ are even integers (due to the symmetry of the four identical nuclei). The radial coefficients $V_{S}^{\lambda_{a} \lambda_{b} \lambda}(R)$ were obtained by means of quadratures of the supermolecular $a b$ initio energies over the angular variables, obtaining a total of 29 coefficients for the quintet PES and 27 for the singlet and triplet ones. The PESs are extended asymptotically ( $R>19$ bohrs) using analytical functions (common to the three multiplicities) based on high level $a b$ initio calculations of electrostatic, dispersion, and induction long-range coefficients. ${ }^{33}$ In Sec. IV, we present a comparison with calculations using the Perugia PES, ${ }^{26}$ which comprises just four radial terms (for each multiplicity) derived from a multiproperty fitting analysis. To give a flavor of the similarities/differences between the two PES considered, we present in Fig. 2 the dependence with the intermolecular distance of the potential matrix elements among the lfs state $|3,3\rangle$ and the (one spin flipping) relaxation channel $|3,1\rangle$. These matrix elements are relevant to the mechanisms proposed by Krems and Dalgarno ${ }^{23}$ and by Volpi and Bohn. ${ }^{24}$ Note that for initial states approaching in an $s$ wave, conservation of $M$ forbids $s$ waves in the spin relaxation channels [see Eq. (6) and Ref. 24]. It can be seen that there are some quantitative differences in the coupling as well as in the long-range behavior. A comparison of properties related to the van der Waals (vdW) coefficient $C_{6}^{000}$ is summarized in Table II.

Cross sections are computed using the code developed by Tscherbul et al., ${ }^{25}$ modified by us to include the hybrid log-derivative/Airy propagator of Alexander and Manolopoulos. $^{40}$ Related routines were taken from the MOLSCAT code. ${ }^{44}$ In this way, the log-derivative propagator of Manolopoulos ${ }^{39}$ is used in the strongly coupled region
TABLE II. Parameters associated with the long-range behavior of the $a b$ initio and Perugia potentials: isotropic vdW coefficient $C_{6}^{000}$, scale length $\left(R_{\mathrm{vdW}}\right)$, and energy $\left(E_{\mathrm{vdW}}\right)$ of the analytical vdW theory (Ref. 46$)$, and height of the $d$-wave centrifugal barrier. $B_{\min }$ is the critical magnetic field for which the $|3,3\rangle-|3,1\rangle$ Zeeman splitting becomes larger than the $d$-wave barrier.

\begin{tabular}{lcc}
\hline \hline & ab initio & Perugia \\
\hline$C_{6}^{000}($ a.u. $)$ & 62.39 & 88.70 \\
$R_{\mathrm{vdW}}\left(a_{0}\right)$ & 22.17 & 24.21 \\
$E_{\mathrm{vdW}}(\mathrm{mK})$ & 10.4 & 8.7 \\
$E_{0}(l=2)(\mathrm{mK})$ & 14.7 & 12.3 \\
$B_{\min }(\mathrm{G})$ & 55 & 46 \\
\hline \hline
\end{tabular}

(from 4.5 to $\left.40.8 a_{0}\right)$ with a fixed short step $\left(0.04 a_{0}\right)$, whereas the Airy propagator of Ref. 40 is used for the long-range region (from 40.8 to 202. $a_{0}$ ) with a variable step size (the ratio between adjacent step sizes being of 1.05). Comparing with the original code of Tscherbul et al., where only the logderivative propagator was used, we found that the errors are less than $0.5 \%$ while the new propagation is about ten times faster due to the smaller number of integration steps as well as the use of the computationally less expensive Airy propagator.

The total wavefunction is expanded using a basis set comprising three rotational levels $\left(n_{a}, n_{b}=0,2,4\right)$ and four partial waves $(l=0,2,4,6)$, equal to that employed in Ref. 25. Although exact positions of the resonances might change with an increase of the basis size, this basis is sufficient to retrieve the main features of the collision dynamics (see, e.g., Ref. 36 and references therein for a discussion on the sensitivity to basis set size in the ultracold regime). Regarding the convergence of the cross sections with the projection of the total angular momentum, $M$, it is found that for translational energies lower than $10^{-4} \mathrm{~K}$, just the $M=2$ block calculation is sufficient, while for larger energies, five blocks ( $M=0-4)$ have to be summed up. Typical run times are about $18 \mathrm{~h}$ for a given value of $M$, energy and magnetic field.

\section{RESULTS AND DISCUSSION}

We present first the results concerning the magnetic-field dependence at very low energies and, in a subsequent section, we report those related to the translational energy dependence, including the transition from the ultracold to the cold regimes.

\section{A. Magnetic-field dependence at $1 \mu \mathrm{K}$}

The magnetic-field dependence of the cross sections for the $l f s$ state $|3,3\rangle$ at $1 \mu \mathrm{K}$ is summarized in Fig. 3 [panels (a) and (b)], where results obtained using the $a b$ initio and Perugia PES are compared. In Fig. 3(c) we report the elasticto-inelastic ratio, $\gamma$, more specifically, the ratio between the elastic cross section and those inelastic ones leading to untrapped states: $\left|\zeta_{a}^{\prime}, \zeta_{b}^{\prime}\right\rangle=|3,1\rangle,|2,2\rangle,|2,1\rangle$, and $|1,1\rangle$. Note that new calculations with the Perugia PES were performed using the same basis set as with the $a b$ initio PES (there are 

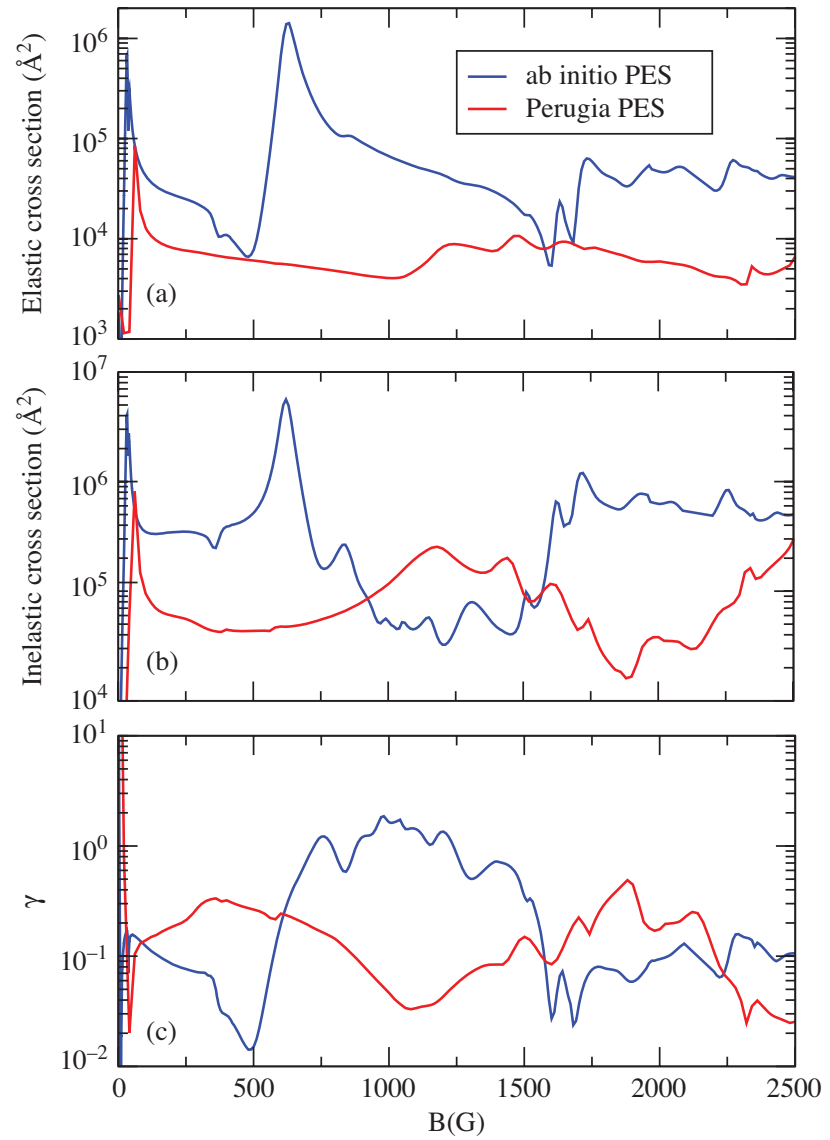

FIG. 3. Magnetic-field dependence for collisions of ${ }^{17} \mathrm{O}_{2}+{ }^{17} \mathrm{O}_{2}$ in the initial lfs state $|3,3\rangle$ and translational energy of $1 \mu \mathrm{K}$. (a) Elastic cross sections; (b) total inelastic cross sections; and (c) ratio $\gamma$ between elastic and inelastic (untrapping) cross sections. Blue and red colors correspond to using the ab initio (Ref. 32) and the Perugia (Ref. 26) PESs, respectively.

some quantitative changes between present calculations and those given in Fig. 3 of Ref. 25 where a smaller basis was employed). There are various noticeable differences between the two PESs. On the one hand, elastic and inelastic cross sections calculated with the $a b$ initio PES are much larger than those using the Perugia PES and, in addition, they exhibit more marked Feshbach resonance structures [Figs. 3(a) and 3(b)]. On the other hand, although there are large variations of the elastic-to-inelastic ratio with the magnetic field, it can be seen that both PESs produce values which, on average, are of the same order of magnitude. The cases of very low fields $(B<50 \mathrm{G})$, where $\gamma$ is much larger for the Perugia PES, and around $1000 \mathrm{G}$, where the value from the ab initio PES becomes very large, are discussed in more detail below.

We discuss first the background behavior of the cross sections of Fig. 3. The elastic cross sections correspond to a background scattering length, $a_{b g}$, of about 118 and $32 a_{0}$, for the $a b$ initio and Perugia PESs, respectively ( $a_{b g}$ is the modulus of the complex scattering length ${ }^{45}$ ). These quantities are larger than the scattering lenghts purely due to the vdW potential, ${ }^{30}$ $\bar{a}$, of 22 and $24 a_{0}$, respectively. The particularly large value of the elastic cross section using the ab initio PES can be explained by existence of a close quasibound state varying with magnetic field at the same rate than the entrance channel. Re- garding inelastic cross sections, the one from the $a b$ initio PES is on average about ten times larger than the result corresponding to the Perugia PES. This difference can be qualitatively rationalized by resorting to the analytic van der Waals theory, ${ }^{46,47}$ which takes the solutions of the vdW potential ${ }^{48}$ as the reference for the multichannel quantum defect theory. ${ }^{49}$ A key parameter in that approach is the short-range squared amplitude of the entrance channel wavefunction, which near threshold is proportional to ${ }^{46}$

$$
\lim _{k_{0} \rightarrow 0} C_{b g}\left(k_{0}\right)^{-2}=k_{0} \bar{a}\left[1+\left(1-\frac{a_{b g}}{\bar{a}}\right)^{2}\right],
$$

$k_{0}$ being the wavenumber of the incoming channel. Since inelastic cross sections are proportional to $C_{b g}\left(k_{0}\right)^{-2},{ }^{49} \mathrm{Eq}$. (14) implies that the value of $a_{b g}$ affects the threshold behavior of the inelastic cross sections. It follows, then, that the very large inelastic cross sections calculated with the $a b$ initio PES (compared to those corresponding to the Perugia PES) are explained by the magnitude of the corresponding background scattering length. Within this framework, one can expect that the elastic-to-inelastic ratio becomes less sensitive to $a_{b g}$ than the cross sections themselves, since both elastic and inelastic cross sections are approximately proportional to $a_{b g}^{2}$. This is the result of Fig. 3(c), where the average value of $\gamma$ is about the same for both potentials.

We now turn to discuss the resonant structures of Fig. 3. At this point, it is convenient to mention the work of Hutson ${ }^{31}$ who analyzed the threshold behavior of Feshbach resonances in the presence of inelastic scattering. He found that-in contrast to the case of a pure elastic scattering-resonance peaks may be significantly suppressed and, in this way, the collisional process may become insensitive to the details of the potential. With this in mind, the profiles obtained in Fig. 3 are rather unexpected given the considerable anisotropy of the $\mathrm{O}_{2}-\mathrm{O}_{2}$ interaction. In connection with this issue, let us digress for a while and study the resonance patterns for a purely elastic scattering event, as is the case of the magnetic-field dependence of the lowest high-field-seeking ( $h f s$ ) state $|1,1\rangle$ (see Fig. 1). The result for the ab initio PES at $1 \mu \mathrm{K}$, using a reduced basis $\left(n_{\max }=4, l_{\max }=4\right)$, is shown in Fig. 4 and can be directly compared with Fig. 4 of Ref. 25. For both PESs, a high density of very pronounced resonances is obtained. For the $a b$ initio PES there is a slightly larger number of peaks, and some of them are wider. Also, the baseline of the cross section computed using the $a b$ initio PES is much larger than that using the Perugia PES, as occurs for the $l f s$ state. A similar density of quasibound states is expected when the entrance channel is the lf $s$ state but presence of inelastic channels substantially modify the resonance lineshapes. ${ }^{31}$ To show this, it is convenient to write down the behavior of the $S$ matrix in the neighborhood of an isolated resonance, ${ }^{31,50}$

$$
S_{j k}(E)=S_{j k}^{b g}-i \frac{g_{E j} g_{E k}}{E-E_{r}+i \Gamma_{E} / 2},
$$

where $k$ and $j$ are the incoming and outgoing channels, respectively, $S_{j k}^{b g}$ is the background $S$ matrix, $E$ is the total energy, $E_{r}$ is the resonance position, $\Gamma_{E}$ is the resonance width, and (complex) $g_{E i}$ involve couplings between resonance and channel $i$ wavefunctions, ${ }^{51}$ such that the partial width for 


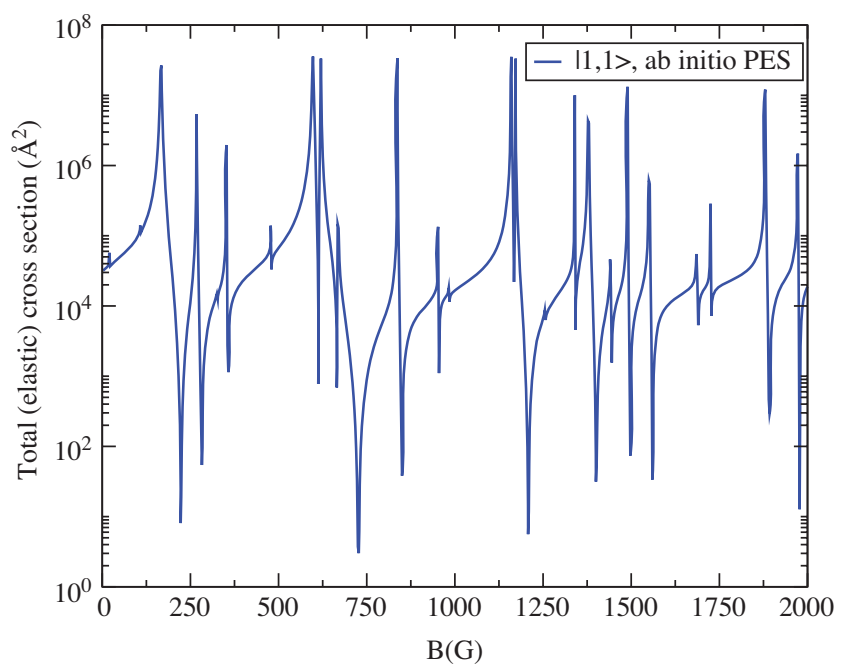

FIG. 4. Total cross section vs magnetic-field for the $h f s$ state $|1,1\rangle$ at a translational energy of $1 \mu \mathrm{K}$ and using the ab initio PES. Note that only the elastic channel is open. The result can be compared with Fig. 4 of Ref. 25, corresponding to the Perugia PES.

channel $i$ is given as $\Gamma_{E i}=\left|g_{E i}\right|^{2}$ and $\Gamma_{E}=\sum_{i} \Gamma_{E i}$. A key point in Hutson's argument is that $g_{E k}$ elements are proportional to the square root of the incoming channel wavenumber $k_{0}^{1 / 2}$. Then, as $k_{0}$ decreases and if the resonant state is also coupled to inelastic channels, the radius of the circle described by $S_{j k}$ drops to zero and peaks in cross sections become significantly suppressed. ${ }^{31}$ The analytical vdW theory gives a more detailed threshold behavior of the $g_{E k}$ elements, as they become proportional to the square root of Eq. (14). Hence, if $a_{b g}$ is sufficiently large, $g_{E k}$ will tend to its threshold value (zero) rather slowly, and as a consequence, more pronounced peaks in the cross sections can be obtained. This explains why we find a marked resonance structure, especially for the ab initio PES. Nevertheless, as noted in Ref. 31 , a relatively large ratio between elastic and inelastic partial widths is also needed in order to obtain pronounced resonance profiles. It is reasonable to expect that, among all the quasibound states that should be crossing the $l f s$ state, only some of them will have particularly large elastic partial widths, so only a few marked resonance features will "survive," as in fact it occurs (Fig. 3).

We have just seen that a large $a_{b g}$ enhances the shortrange couplings between the resonance and the incoming wavefunctions. In this situation the dynamics must become very sensitive to the short-range region of the potential. In order to study the role played by the short-range versus the long-range features of the intermolecular potential, we have performed a test calculation where the long-range anisotropy of the potential is switched off. To this end, the ab initio PES has been modified by imposing, for $R>19 a_{0}$, an exponential decay of all radial terms of Eq. (13) except the isotropic one $\left(\lambda_{a}, \lambda_{b}, \lambda\right)=(000)$. The new cross sections are compared with those corresponding to the correct long-range behavior in Fig. 5. This figure clearly shows that the resonance structure is rather insensitive to the long-range anisotropy of the interaction and, therefore, short-range couplings must be playing a dominant role.

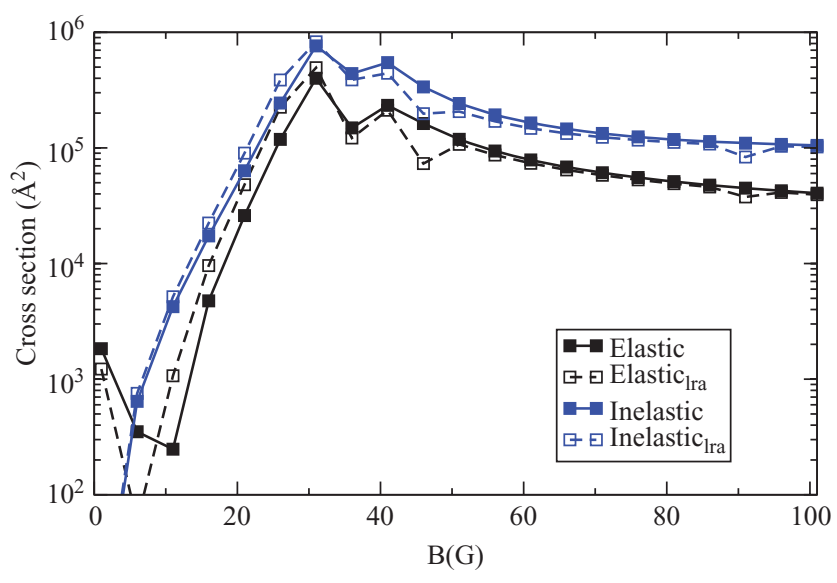

FIG. 5. Effect of the long-range anisotropy of the ab initio PES: Magneticfield dependence of elastic and total inelastic cross sections for the lfs state $|3,3\rangle$ at $10 \mu \mathrm{K}$. Thick lines joined by filled squares show results using the correct long-range anisotropy (Refs. 32 and 33) while dashed lines joined by open squares correspond to calculations where the long-range anisotropy of the interaction has been switched off.

Finally, it is interesting to note from Fig. 3(b) that, for the $a b$ initio PES, there is a significant suppression of inelastic scattering for magnetic fields ranging from 750 to $1500 \mathrm{G}$. This feature must be related with the prominent resonance at about $600 \mathrm{G}$ and it must be due to interferences between the background and resonant $S$ matrices leading to asymmetric line-shapes of the state-to-state cross sections. ${ }^{52}$ Note that this reduction entails a considerable increase of the ratio $\gamma$ for a wide range of magnetic fields. A similar behavior (with an even larger suppression of inelastic scattering) has been found in ${ }^{4} \mathrm{He}+{ }^{16} \mathrm{O}_{2}$ magnetic Feshbach resonances. ${ }^{22}$

Analogously, it is also worth mentioning that, for the results corresponding to the $a b$ initio PES, the elastic scattering on the left-hand-side of the resonance at about $B=30$ $\mathrm{G}$ is suppressed. This feature, already present in Fig. 3, can be more clearly seen in Fig. 5, where the elastic cross section becomes very small around $10 \mathrm{G}$. In this case, the corresponding ratio $\gamma$ becomes much smaller than expected (from the well known effect of suppresion inelastic scattering due to centrifugal barriers ${ }^{24,25,28}$ ).

\section{B. Translational energy dependence}

In Fig. 6, dependence of the cross sections with kinetic energy is given for several selected values of the magnetic field. In agreement with predictions based on the analytical $\mathrm{vdW}$ theory, ${ }^{46}$ two very different regimes are noticed for energies larger or smaller than $E_{v d W} \approx 10 \mathrm{mK}$ (see Table II). For the higher energy range, elastic and inelastic cross sections exhibit a weak dependence with the field, the ones obtained using the Perugia PES being larger than their $a b$ initio counterparts, in consistency with previous studies at higher energies. ${ }^{41}$ For energies lower than the crossover $\left(E_{\mathrm{vdW}}\right)$, cross sections become more dependent on the magnetic field. This is mainly due to the effect of the resonances in the ultracold regime, but in the case of the Perugia PES, suppression of inelastic cross sections at low fields (due to the centrifugal barriers $^{25,28}$ ) also plays a role. 

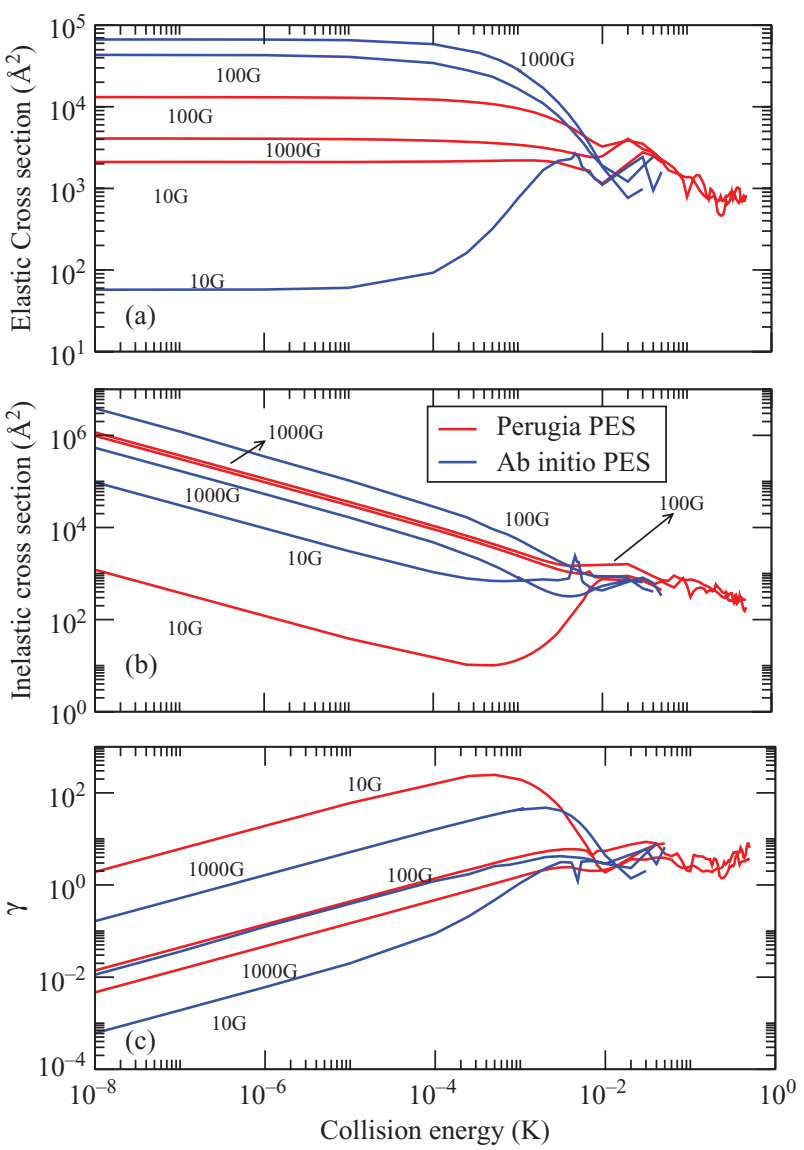

FIG. 6. Translational energy dependence of the collisional processes for the Ifs state $|3,3\rangle$ : a comparison between ab initio and Perugia PESs for different values of magnetic field. (a) Elastic cross sections; (b) Total inelastic cross sections; and (c) Elastic-to-inelastic ratio $\gamma$.

It is interesting to highlight that a relatively high value of the elastic-to-inelastic ratio has been obtained between 1 and $10 \mathrm{mK}$ in the calculation using the ab initio PES at $1000 \mathrm{G}$ [Fig. 6(c)]. This result is related to the asymmetry of the lineshape and the suppression of spin-changing processes on the right-hand-side of the resonance at $600 \mathrm{G}$ and $1 \mu \mathrm{K}$, discussed above (Fig. 3).

A more detailed study of the cross sections calculated with the $a b$ initio PES for low values of the field $(B \leq 50 \mathrm{G})$ is given in Fig. 7. An impressive dependence with $B$ is noticed for energies just below $10 \mathrm{mK}$. Between 1 and $10 \mathrm{mK}$, complicated resonance structures are seen which are particularly acute for the elastic cross section. These features are related to the prominent resonance around $30 \mathrm{G}$ at much lower energies (reported in Fig. 3 and more clearly seen in Fig. 5). In other words, they are expressions - at several different energies and magnetic fields - of the same quasibound state. For instance, note the resemblance between the asymmetric line shapes of the elastic cross section at $B=1$ and $5 \mathrm{G}$ and between 1 and $10 \mathrm{mK}$ [Fig. 7(a)], with the magnetic-field dependence at much lower energies for fields $B<30 \mathrm{G}$, as shown in Fig. 5 . A detailed tracking of these resonances would involve nontrivial lineshape fittings and has not been attempted here. On the other hand, it should be noted that, for the range of magnetic fields of Fig. 7 and up to translational energies of at least
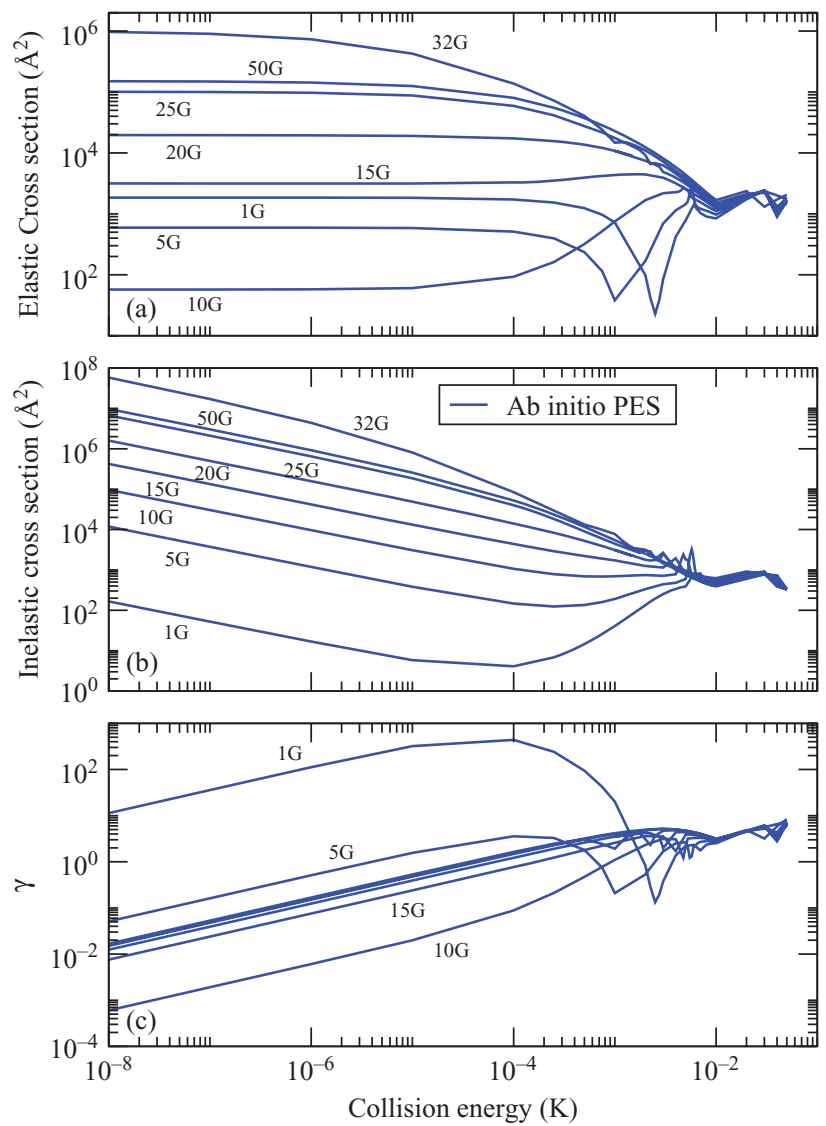

FIG. 7. Same as Fig. 6 but for different values of magnetic field near the $32 \mathrm{G}$ resonance of the calculations with the $a b$ initio PES.

$1 \mathrm{mK}$, spin-changing collisions should be suppressed due to existence of centrifugal barriers in all outgoing channels. ${ }^{25,28}$ In Fig. 7(b) it can be seen that, except for the lowest value of $B$ $(1 \mathrm{G})$, such a suppression does not occur, in contrast with the results using the Perugia PES [see Ref. 25 and Fig. 6(b)]. This must be due to a significant tunneling through the centrifugal barriers for energies/fields close to the resonance. Consequently, the ratios $\gamma$ are particularly small for this range of fields [Fig. 7(b)].

A further analysis of the sensitivity of the elastic-toinelastic ratio to the details of the PES has been performed. We have artificially modified the anisotropy of the present $a b$ initio PES by multiplying all the terms in the spherical harmonic expansion [Eq. (13)] - except the isotropic onesby a factor $\beta$ ranging form 0.98 to 1.02 . In Fig. 8 we show the results for different translational energies and magnetic fields. It can be seen that, while for $20 \mathrm{mK}$ there is not a strong variation of $\gamma$ with $\beta$, for lower energies $(1 \mathrm{mK}$ and $1 \mu \mathrm{K})$, this ratio changes tremendously with the anisotropy of the potential. In the new calculations ( $\beta=0.98$ and 1.02$)$, no nearby resonances appear for the energies/fields considered and hence, results are "more standard," i.e., very large values of $\gamma$ are now attained for low values of the field $(B<50 \mathrm{G})$, in agreement with the expected suppression of inelastic scattering, but smaller $\gamma$ 's are obtained for $B=1000$ G. However, note that, contrarily to a first order perturbation theory, the largest ratios are obtained with the most anisotropic PES $(\beta=1.02)$. 

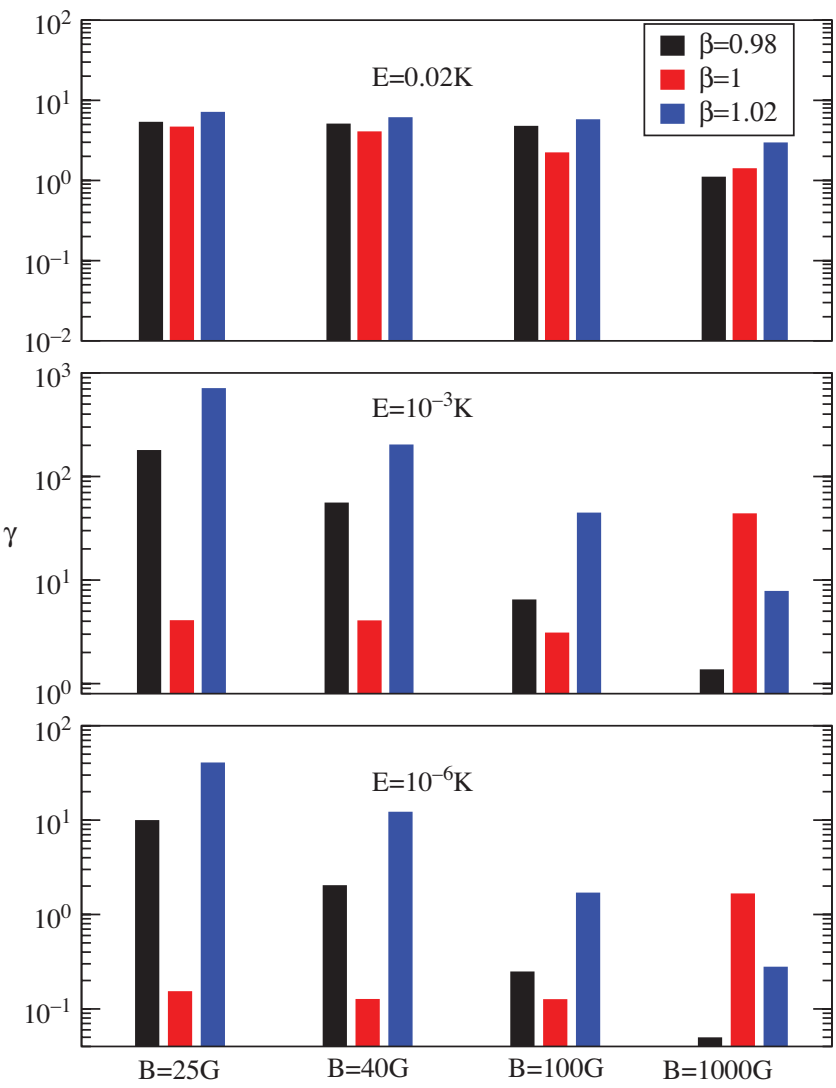

FIG. 8. Study of the sensitivity of the elastic-to-inelastic ratio $\gamma$ to the anisotropy of the $a b$ initio PES. All terms of the spherical harmonic expansion are multiplied by $\beta$ except the isotropic one. The effect is shown for different translational energies and magnetic fields.

\section{CONCLUDING DISCUSSION}

We have performed a detailed study of cold and ultracold molecule-molecule collisions in the presence of a magnetic field for a system with a significant anisotropy such as $\mathrm{O}_{2}+\mathrm{O}_{2}$. A thorough comparison has been made between a high quality $a b$ initio PES and previous studies ${ }^{25}$ where a different PES was used. Several interesting findings have emerged from this approach regarding the anisotropy as well as the relative influence of long and short components of the interaction. For the $a b$ initio PES, a large background scattering length gives rise to pronounced resonance structures in the ultracold regime (translational energies $<10 \mathrm{mK}$ ). As a consequence, the ratio between elastic and inelastic cross sections, $\gamma$, is very dependent on the magnetic field as well as on the short-range anisotropy of the PES. Therefore, quantitative predictions for this important parameter become rather risky. However, and as a general trend, we can indicate that high values of $\gamma$ could be achieved in the vicinity of asymmetric Fano resonances, or for low fields, $B<50 \mathrm{G}$. Note that the maximum temperature that can be held in a trap with such a depth would be of about $1 \mathrm{mK} .{ }^{25,53}$

A key issue is the large density of quasibound states of the $\mathrm{O}_{2}+\mathrm{O}_{2}$ system, best illustrated in the magneticfield dependence of the elastic cross sections of the lowest high-field-seeking state. In view of this, having obtained a large background scattering length does not seem a rare event. Present behavior might be characteristic of a range of molecule-molecule systems as well, that is to say, as the number of degrees of freedom increases, a larger density of quasibound states, including near threshold resonances, can be expected, ${ }^{54}$ which in turn makes dynamics richer. Very recently, Suleimanov and $\mathrm{Krems}^{55}$ have proposed an efficient method for locating Feshbach resonances in external fields. The new method could be very useful for the comparison of spectral patterns obtained from different potentials or between different molecular systems.

\section{ACKNOWLEDGMENTS}

We are indebted to Roman V. Krems for encouragement and for giving us essential insight along several stages of this work. We wish to thank M. H. Alexander, D. E. Manolopoulos, and J. M. Hutson for the use of the Hybrid Propagator routines of the MOLSCAT code, and to M. Bartolomei, E. Carmona-Novillo, and R. HernándezLamoneda for the use of the $a b$ initio PES. J.P.-R. acknowledges hospitality in the Departmtent of Chemistry of UBC (Canada) and support from a predoctoral JAE CSIC grant. The work has been funded by Ministerio de Ciencia e Innovación (Spain, Grant Nos. CTQ2007-62898-BQU and FIS2010-22064-C02-02). We also thank CESGA (Spain) for allocation of computing time.

${ }^{1}$ A. André, D. DeMille, J. M. Doyle, M. D. Lukin, S. E. Maxwell, P. Rabl, R. J. Schoelkopf, and P. Zoller, Nat. Phys. 2, 636 (2006).

${ }^{2}$ B. L. Lev, R. E. Meyer, E. R. Hudson, B. C. Sawyer, J. L. Bohn, and J. Ye, Phys. Rev. A 74, 061402 (2006).

${ }^{3}$ R. V. Krems, Phys. Chem. Phys. Chem. 10, 4079 (2008).

${ }^{4}$ M. T. Bell and T. P. Softley, Mol. Phys. 107, 99 (2009).

${ }^{5}$ F. A. Gianturco and M. Tacconi, Faraday Discuss. 142, 463 (2009).

${ }^{6}$ D. W. Wang, M. D. Lukin, and E. Demler, Phys. Rev. Lett. 97, 180413 (2006).

${ }^{7}$ A. Micheli, K. G. Brennen, and P. Zoller, Nat. Phys. 2, 341 (2006).

${ }^{8}$ H. P. Büchler, A. Micheli, and P. Zoller, Nat. Phys. 3, 726 (2007).

${ }^{9}$ D. DeMille, Phys. Rev. Lett. 88, 067901 (2002).

${ }^{10}$ P. Rabl, D. DeMille, J. M. Doyle, M. D. Lukin, R. J. Schoelkopf, and P. Zoller, Phys. Rev. Lett. 96, 033003 (2006).

${ }^{11}$ M. G. Moore and A. Vardi, Phys. Rev. Lett. 88, 160402 (2002).

${ }^{12}$ C. J. Pethick and H. Smith, Bose-Einstein Condensation in Dilute Gases (Cambridge University Press, England, 2002).

${ }^{13}$ B. Friedrich and J. M. Doyle, Chem. Phys. Chem. 10, 604 (2009).

${ }^{14}$ E. Narevicius, A. Libson, C. G. Parthey, I. Chavez, J. Narevicius, U. Even, and M. Raizen, Phys. Rev. A 77, 051401 (2008).

${ }^{15}$ R. Fulton, A. I. Bishop, M. N. Shneider, and P. F. Baker, Nat. Phys. 2, 465 (2006).

${ }^{16}$ E. Narevicius, S. T. Bannerman, and M. G. Raizen, New. J. Phys. 11, 055046 (2009).

${ }^{17}$ F. Schreck, G. Ferrari, K. L. Corwin, J. Cubizolles, L. Khaykovich, M.-O. Mewes, and C. Salomon, Phys. Rev. A 64, 011402 (2001).

${ }^{18}$ H. F. Hess, Phys. Rev. B 34, 3476 (1986).

${ }^{19}$ M. H. Anderson, J. R. Ensher, M. R. Mattew, C. E. Wieman, and E. A. Cornell, Science 269, 198 (1995).

${ }^{20}$ K. B. Davis, M. O. Mewes, M. R. Andrews, N. J. V. Druten, D. S. Durfee, D. M. Kurn, and W. Ketterle, Phys. Rev. Lett. 75, 3969 (1995).

${ }^{21}$ C. Chin, R. Grimm, P. Julienne, and E. Tiesinga, Rev. Mod. Phys. 82, 1225 (2010).

${ }^{22}$ J. M. Hutson, M. Beyene, and M. L. González-Martínez, Phys. Rev. Lett. 103, 163201 (2009).

${ }^{23}$ R. V. Krems and A. Dalgarno, J. Chem. Phys. 120, 2296 (2004).

${ }^{24}$ A. Volpi and J. L. Bohn, Phys. Rev. A 65, 052712 (2002).

${ }^{25}$ T. V. Tscherbul, Y. V. Suleimanov, V. Aquilanti, and R. V. Krems, New J. Phys. 11, 055021 (2009). 
${ }^{26}$ V. Aquilanti, D. Ascenzi, M. Bartolomei, D. Cappelletti, S. Cavalli, M. de Castro Vitores, and F. Pirani, J. Am. Chem. Soc. 121, 10794 (1999).

${ }^{27}$ B. Friedrich, R. deCarvalho, J. Kim, D. Patterson, J. D. Weinstein, and J. M. Doyle, J. Chem. Soc., Faraday Trans. 94, 1783 (1998).

${ }^{28}$ A. V. Avdeenkov and J. L. Bohn, Phys. Rev. A 64, 052703 (2001).

${ }^{29}$ D. Patterson and J. M. Doyle, J. Chem. Phys. 126, 154307 (2007).

${ }^{30}$ G. F. Gribakin and V. V. Flambaum, Phys. Rev. A 48, 546 (1993).

${ }^{31}$ J. M. Hutson, New J. Phys. 9, 152 (2007).

${ }^{32}$ M. Bartolomei, E. Carmona-Novillo, M. I. Hernández, J. CamposMartínez, and R. Hernández-Lamoneda, J. Chem. Phys. 133, 124311 (2010).

${ }^{33}$ M. Bartolomei, E. Carmona-Novillo, M. I. Hernández, J. CamposMartínez, and R. Hernández-Lamoneda, J. Comput. Chem. 32, 279 (2011).

${ }^{34}$ J. Pérez-Ríos, G. Tejeda, J. M. Fernández, M. I. Hernández, and S. Montero, "Inelastic collisions in molecular oxygen at low temperature $(4<\mathrm{T}<34 \mathrm{~K})$ : Close-coupling calculations versus experiment," J. Chem. Phys. (submitted).

${ }^{35}$ M. Mizushima, The Theory of Rotating Diatomic Molecules (Wiley, New York, 1975).

${ }^{36}$ L. M.C. Janssen, P. S. Żuchowski, A. van der Avoird, G. C. Groenenboom, and J. M. Hutson, Phys. Rev. A 83, 022713 (2011).

${ }^{37}$ M. T. Hummon, T. V. Tscherbul, J. Klos, H.-I. Lu, E. Tsikata, W. C. Campbell, A. Dalgarno, and J. M. Doyle, Phys. Rev. Lett. 106, 053201 (2011)

${ }^{38}$ E. Tiesinga, B. J. Verhaar, and H. T.C. Stoof, Phys. Rev. A 47, 4114 (1993).
${ }^{39}$ D. E. Manolopoulos, J. Chem. Phys. 85, 6425 (1986).

${ }^{40}$ D. E. Manolopoulos and M. H. Alexander, J. Chem. Phys. 86, 2044 (1987).

${ }^{41}$ J. Pérez-Ríos, M. Bartolomei, J. Campos-Martínez, M. I. Hernández, and R. Hernández-Lamoneda, J. Phys. Chem. A 113, 14952 (2009).

${ }^{42}$ G. Gioumousis and C. F. Curtiss, J. Chem. Phys. 29, 996 (1958).

${ }^{43}$ G. Gazzoli and C. D. Esposti, Chem. Phys. Lett. 113, 501 (1985).

${ }^{44} \mathrm{~J}$. M. Hutson and S. Green, molscat version 14 Collaborative Computational Project No. 6, U.K. Science and Engineering Research Council, 1994.

${ }^{45}$ N. Balakrishnan, V. Kharchenko, R. C. Forrey, and A. Dalgarno, Chem. Phys. Lett. 280, 5 (1997).

${ }^{46}$ P. S. Julienne and B. Gao, Atomic Physics 20, edited by C. Roos, H. Haffner, and R. Blatt (AIP, New York, 2006), p. 261.

${ }^{47}$ B. Gao, Phys. Rev. A 58, 4222 (1998).

${ }^{48}$ B. Gao, Phys. Rev. A 58, 1728 (1998).

${ }^{49}$ P. S. Julienne and F. H. Mies, J. Opt. Soc. Am. B 6, 2257 (1989).

${ }^{50}$ H. Feshbach, Ann. Phys. 5, 357 (1958).

${ }^{51}$ W. H. Miller, J. Chem. Phys. 52, 543 (1970).

${ }^{52}$ U. Fano, Phys. Rev. 124, 1866 (1961).

${ }^{53}$ J. M. Doyle, B. Friedrich, J. Kim, and D. Patterson, Phys. Rev. A 52, 2515 (1995).

${ }^{54}$ J. L. Bohn, A. V. Avdeenkov, and M. P. Deskevich, Phys. Rev. Lett. 89, $203202(2002)$

${ }^{55}$ Y. V. Suleimanov and R. V. Krems, J. Chem. Phys. 134, 014101 (2011). 